\title{
Medical Image of the Week: Renal Infarction
}
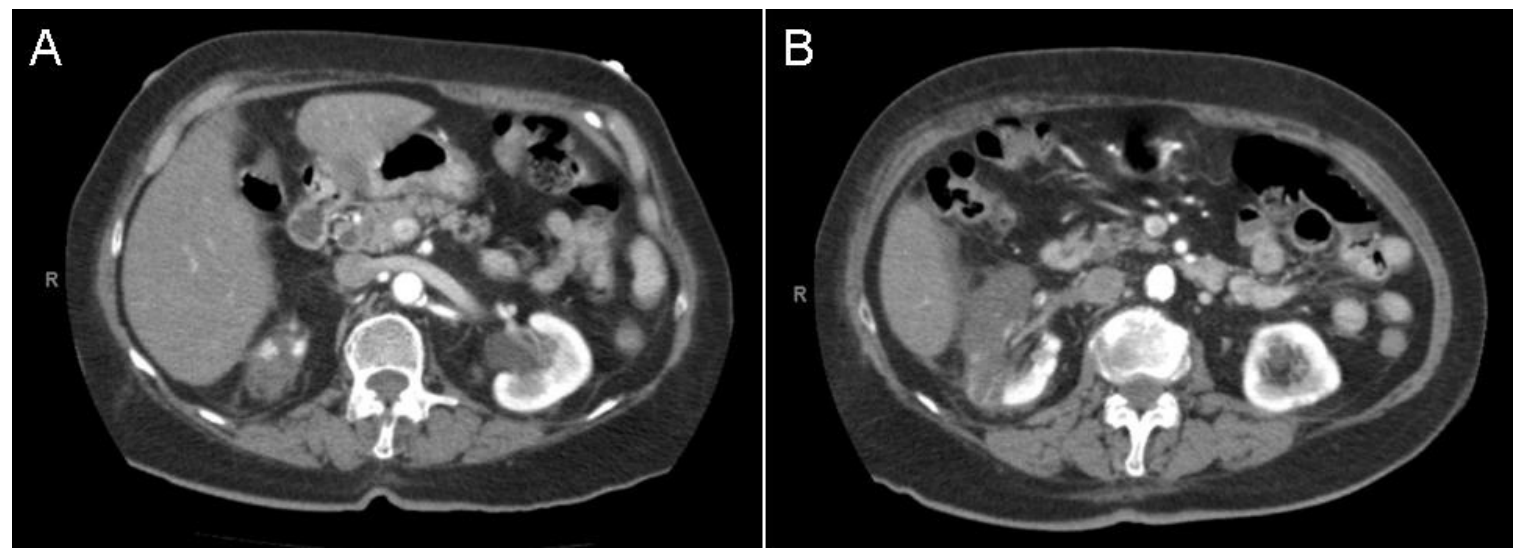

Figure 1. Contrast enhanced CT of the abdomen showing the majority of the right kidney infarcted with some preservation of the superior pole.

A 79-year-old woman with past medical history of persistent atrial fibrillation not on anticoagulation, coronary artery disease, hypertension, diabetes, and hyperlipidemia presented with right flank pain accompanied by nausea and vomiting for two days. Laboratory studies showed leukocytosis with creatinine of 1.2. Urinalysis was negative for signs of infection and red blood cells. However, despite being on analgesic, she continued to have flank pain. The patient subsequent underwent CT scan of the abdomen and pelvis the next day, which showed that the majority of the right kidney was infarcted. Interestingly, there were two right-sided renal arteries and a thrombus was seen in the inferior main right renal artery. The superior pole of the right kidney was preserved as a result of the patent accessory renal artery. Due to delayed presentation of more than 48 hours after onset of pain, the tissue could not be re-vascularized by vascular surgery. Her renal function remained intact and her flank pain gradually improved.

Acute renal infarction is difficult to diagnose as it is mimicked by more commonly seen causes such as pyelonephritis and nephrolithiasis. Pain in the unilateral flank and/or abdomen is the hallmark presenting feature, however nausea, vomiting, and fever are also common. New or increasingly severe hypertension is found in approximately half of acute renal infarction diagnosis, a sign that should raise clinical suspicion in similar clinical scenario. Proteinuria is another feature that may be present on urinalysis. Creatinine elevation consistent with acute kidney injury (AKI) occurs in approximately $30-40 \%$ of cases (1). Leukocytosis is commonly seen in as many as three-quarters of patients with renal infarct. The most common laboratory finding in renal infarction is elevation of $\mathrm{LDH}$, although this is nonspecific and does not necessarily aid in specific diagnosis (2). Diagnosis is usually made through contrast enhanced CT abdomen, however angiography may also be used. 
Our patient also had two renal arteries supplying her right kidney, allowing for the superior pole of her renal parenchyma to be spared and thus her kidney function. The kidney tolerates ischemia for approximately 12 hours, making early diagnosis paramount. In patient's such as the one described here that involves all or majority of one kidney, embolectomy is recommended because of favorable outcomes in prior studies (3). In summary, the challenge of early diagnosis of renal infarction lies in the recognition of nonspecific clinical symptoms and signs in an already rare occurrence. Recognizing these signs within hours of presentation may be the difference between viable renal tissue and death of a kidney. Keeping a high suspicion in patients with atrial fibrillation will also aid in early diagnosis.

Jessica August MD and Jennifer J Huang DO

Department of Internal Medicine

University of Arizona

Tucson, AZ

\section{References}

1. Bae EJ, Hwang K, Jang HN, Kim MJ, Jeon DH, Kim HJ, Cho HS, Chang SH, Park DJ. A retrospective study of short- and long-term effects on renal function after acute renal infarction. Ren Fail. 2014;36(9):1385-9. [CrossRef] [PubMed]

2. Antopolsky M, Simanovsky N, Stalnikowicz R, Salameh S, Hiller N. Renal infarction in the ED: 10-year experience and review of the literature. Am J Emerg Med. 2012;30(7):1055-60. [CrossRef] [PubMed]

3. Tsai SH, Chu SJ, Chen SJ, Fan YM, Chang WC, Wu CP, Hsu CW. Acute renal infarction: a 10-year experience. Int J Clin Pract. 2007;61(1):62-7. [CrossRef] [PubMed] 